\title{
$\alpha$-enolase is highly expressed in liver cancer and promotes cancer cell invasion and metastasis
}

\author{
LIHONG ZHANG $^{1}$, TAO LU ${ }^{1}, \mathrm{YE} \mathrm{YANG}^{2}$ and LIANGFENG HU ${ }^{1}$ \\ Departments of ${ }^{1}$ Clinical Laboratory Center and ${ }^{2}$ Pathology, Shaoxing People's Hospital, \\ Shaoxing, Zhejiang 312000, P.R. China
}

Received August 30, 2019; Accepted March 19, 2020

DOI: $10.3892 / \mathrm{ol} .2020 .12003$

\begin{abstract}
The expression levels of $\alpha$-enolase, also known as enolase 1 (ENO1), in liver cancer tissues and the autoantibody levels of ENO1 in the sera of patients with liver cancer were detected to investigate the function of ENO1 in the invasion and metastasis of liver cancer, as well as its clinical diagnostic value. Small interfering RNA (siRNA) was used to disrupt ENO1 gene expression in HepG2 and Huh7 liver cancer cells. The proliferation ability of liver cancer cells was assessed using Cell Counting Kit-8 (CCK-8); the migration ability of liver cancer cells was assessed using scratch tests; and the migration and invasion abilities of liver cancer cells were assessed using Transwell assays. ENO1 expression in liver cancer tissues $(43.8 \%)$ was significantly higher than that in benign liver lesions $(15.2 \%)(\mathrm{P}=0.005)$. The serum anti-ENO1 antibody levels in the liver cancer group were significantly higher than those in the control and benign liver lesion groups $(\mathrm{P}<0.001)$. After ENO1 gene interference, the proliferation, migration and invasion abilities of HepG2 and Huh7 liver cancer cells exhibited different degrees of suppression. The results revealed that ENO1 promotes liver cancer invasion and metastasis; ENO1 plays an important role in liver cancer and can be used as a potential liver cancer-associated marker.
\end{abstract}

\section{Introduction}

Liver cancer is one of the most common malignant tumours in clinical practice (1). There are currently $\sim 800,000$ new liver cancer cases every year worldwide, and almost half of these cases are in China (2). Liver cancer has an insidious onset, and is prone to invasion and metastasis. At diagnosis, surgery is no longer a treatment option for the majority of patients; therefore, the mortality is very high. The early diagnosis and

Correspondence to: Dr Liangfeng $\mathrm{Hu}$, Department of Clinical Laboratory Center, Shaoxing People's Hospital, 568 Zhongxing North Road, Shaoxing, Zhejiang 312000, P.R. China

E-mail: 13757586808@163.com

Key words: $\alpha$-enolase, liver cancer, invasion, metastasis, diagnostic value treatment are key for increasing the survival rate of patients with liver cancer (3). Currently, commonly used liver cancer markers, such as $\alpha$ fetoprotein, have a certain specificity; however, the majority of markers have low sensitivity. Therefore, when liver cancer is confirmed, it is often already at a late stage $(4,5)$. Thus, the elucidation of the mechanism underlying liver cancer invasion and metastasis, and the development of highly effective and non-invasive diagnostic and prognostic markers are of great importance for the diagnosis and treatment of liver cancer.

$\alpha$-enolase, also known as enolase 1 (ENO1), is a multifunctional protein. In addition to catalysing glycolysis, ENO1 is also involved in transcription, apoptosis regulation and cell differentiation, and plays an important role in a number of biological and pathophysiological processes. Some studies have reported that ENO1 plays important roles in the development and progression of some malignant tumours (6-8). It has been confirmed that ENO1 is closely associated with liver cancer (9); however, the role of ENO1 in liver cancer is still unclear, and relevant studies on the level of serum anti-ENO1 antibody have not been reported yet. In the present study, the role of ENO1 in the invasion and metastasis of liver cancer cells was investigated, as well as the expression of ENO1 in liver cancer tissues and that of anti-ENO1 antibody in the peripheral blood of patients with liver cancer, to evaluate the clinical value of ENO1 in liver cancer.

\section{Patients and methods}

Clinical specimens. A total of 80 pathological tissue specimens and 56 serum specimens were collected from patients who were pathologically diagnosed with liver cancer and did not receive treatment between January 2017 and June 2019 at Shaoxing People's Hospital (Shaoxing, China). A total of 33 pathological tissue specimens (including 21 males and 12 females; mean age of 62.3 years; 14 cases of liver cyst, 10 cases of liver fibroma, 7 cases of liver abscess, 1 case of hepatitis and 1 case of hepatic hamartoma) and 29 serum specimens (including 16 males and 13 females; mean age of 56.7 years; 13 cases of liver cyst, 10 cases of liver fibroma and 6 cases of liver abscess) were collected from patients with benign liver lesions. In addition, 40 serum specimens from healthy individuals who received a physical examination during the same period were collected and used as the 
control group. A patient flow diagram showing the selection of the study population is presented in Fig. 1. The study was approved by the Ethics Committee of Shaoxing People's Hospital (Shaoxing, China) and signed informed consent was obtained from the patients.

The tissue specimens were fixed in $10 \%$ neutral formalin at room temperature for $24 \mathrm{~h}$, routinely embedded in paraffin, sectioned into $4-\mu \mathrm{m}$-thick sections, and stored at $4^{\circ} \mathrm{C}$. Peripheral blood specimens $(3 \mathrm{ml})$ were placed in vacuum blood collection tubes and centrifuged at $2,000 \mathrm{xg}$ at $4^{\circ} \mathrm{C}$ for $5 \mathrm{~min}$. Serum samples were collected, aliquoted and stored at $-20^{\circ} \mathrm{C}$.

Cells and reagents. The human liver cancer HepG2 and Huh7 cell lines were purchased from the Chinese Academy of Sciences Shanghai Cell Bank. Rabbit anti-ENO1 monoclonal antibodies were purchased from Abcam (cat. no. ab85086) for use in immunohistochemistry and western blotting. Anti-rabbit IgG secondary antibody (cat. no. IB000086) was purchased from OriGene Technologies, Inc., for use in immunohistochemistry. Mouse anti- $\beta$-actin monoclonal antibody (cat. no. DW9562; Bioworld Technology, Inc.), horseradish peroxidase (HRP)-conjugated goat anti-rabbit IgG (cat. no. 130321; Hangzhou HuaAn Biotechnology Co., Ltd.) and anti-mouse antibodies (cat. no. BS12471; Bioworld Technology, Inc.) were used for western blotting. The immunohistochemistry reagent kit was purchased from Fuzhou Maixin Biotech Co., Ltd., (cat. no. KIT5003). The anti-ENO1 antibody ELISA detection reagent kit (cat. no. CSB-EQ027775HU) was purchased from CUSABIO Technology LLC. Small interfering RNA (siRNA) sequences were purchased from Guangzhou RiboBio Co., Ltd., (cat. nos. siG10623162542, siG10623162557 and siN05815122147). Lipo6000 ${ }^{\mathrm{TM}}$ transfection reagent and Cell Counting Kit-8 (CCK-8) were purchased from Beyotime Institute of Biotechnology (cat. nos. C0526 and C0038, respectively). The primers (cat. no. H001) were purchased from Sangon Biotech Co., Ltd. One Step SYBR ${ }^{\circledR}$ PrimeScript $^{\mathrm{TM}}$ RT-PCR kit was purchased from Takara Bio, Inc. (cat. no. RR086A). SDS-PAGE Gel kit (cat. no. P1200) and Phenylmethylsulfonyl fluoride (PMSF; cat. no. P0100) were purchased from Beijing Solarbio Science \& Technology Co., Ltd. Biolonase was purchased from Shanghai Biolong Biotech Co., Ltd. RIPA protein lysis solution was purchased from Biotium, Inc. Protein marker was purchased from Invitrogen (cat. no. 26616; Thermo Fisher Scientific, Inc.). Transwell plates were purchased from Corning, Inc. (cat. no. 3422). Matrigel was purchased from BD Biosciences (cat. no. 356234).

\section{Experimental procedures}

Detection of ENOI levels in liver cancer tissues using immunohistochemistry. The rabbit monoclonal antibody anti-ENO1 was used for analysis. Endogenous peroxidase was blocked with $3 \% \mathrm{H}_{2} \mathrm{O}_{2}$ at room temperature for $10 \mathrm{~min}$ after antigen retrieval in a microwave heated to $121^{\circ} \mathrm{C}$ for $150 \mathrm{sec}$, xylene washing and rehydration in a descending alcohol series (100, 95 and 85\%). The slides were incubated with $10 \%$ normal goat serum (cat. no. ZLI-9056; OriGene Technologies, Inc.) at room temperature for $1 \mathrm{~h}$, followed by three washes using PBS and incubation with anti-ENO1 antibody $(1: 800)$ at $4{ }^{\circ} \mathrm{C}$ overnight. Subsequently, the slides were incubated with anti-rabbit IgG (1:1; cat. no. IB000086; OriGene Technologies, Inc.) at room temperature for $20 \mathrm{~min}$ and stained with 3,3'-diaminobenzidine (1:20; cat. no. PV-8000D; OriGene Technologies, Inc.) for $8 \mathrm{~min}$ at room temperature. Counterstaining was performed with haematoxylin for $20 \mathrm{sec}$ at room temperature.

Five representative fields of each section were selected and captured under a high-power light microscope (magnification, x100; cat. no. BX41; Olympus Corp.), and 100 tumour cells in each field were counted. A total of 500 cells were counted. The localization of ENO1 was observed in the cytoplasm, cell membrane or cell nucleus. Positive cells exhibited yellow or yellow-brown particles. The cells in which the positive signal was $\geq 5 \%$ were considered positive, and those in which the positive signal was $<5 \%$ were considered negative.

Detection of serum anti-ENOI antibodies using ELISA. Standard and patient samples were added to 96-well plates separately, and then $100 \mu \mathrm{l}$ HRP-labelled monoclonal antibody (1:100; included in the anti-ENO1 antibody ELISA detection reagent kit) was added to each well. The plates were washed 5 times after incubation at $37^{\circ} \mathrm{C}$ for $60 \mathrm{~min}$, and then developer A and B (50 $\mu \mathrm{l}$ each) was added to each well. The optical density (OD) values were measured at a $450-\mathrm{nm}$ wavelength after development at $37^{\circ} \mathrm{C}$ for 15 min without light. The concentrations of the standard samples and the measured OD values of each plate were used to plot a standard curve, and a linear regression equation of the standard curve was calculated. The OD values of the samples were introduced into the equation to calculate the concentrations of the samples. Experiments were repeated at least three times.

Cell culture and transfection. Human liver cancer cell lines, HepG2 and Huh7, were routinely cultured at $37^{\circ} \mathrm{C}$ and $5 \% \mathrm{CO}_{2}$ in a humidified environment in complete Dulbecco's modified Eagle's medium (DMEM) containing 10\% foetal bovine serum (FBS) (both HyClone; Thermo Fisher Scientific, Inc.). The culture medium was then aspirated, the cells were washed twice with reduced serum medium (Opti-MEM; Gibco; Thermo Fisher Scientific, Inc.), and $2 \mathrm{ml}$ culture medium was slowly added to each well in the 6-well plates along the inner wall. Next, $250 \mu \mathrm{l} 0.05 \mu \mathrm{M}$ siRNA and negative control (NC) reagent was added to each well and cultured for 4-6 $\mathrm{h}$. The culture medium and the transfection reagent mixture solution were discarded, and the cells were washed twice with PBS. Complete DMEM containing 10\% FBS was added, and the cells were cultured for 24 or $48 \mathrm{~h}$ in a $37^{\circ} \mathrm{C}$ and $5 \% \mathrm{CO}_{2}$ humidified environment. The cells were collected, and RNA and protein were extracted to determine the interference effect. The sequence of siRNA specific to ENO1 was different in the interference group 1 (si-1) (5'-GCATTGGAGCAGAGGTTTA-3') and the interference group 2 (si-2) (5'-CCCAGTGGTGTCTATCGAA-3').

Validation of the interference effect. At $48 \mathrm{~h}$ post-transfection, the interference effect was measured. The interference effect of ENO1-siRNA was evaluated by determining the downregulation of the ENO1 gene and protein. Reverse transcription-quantitative PCR (RT-qPCR) and western blot analysis were performed 3 times. The mean value of the experimental results was used as the relative expression level of ENO1. 


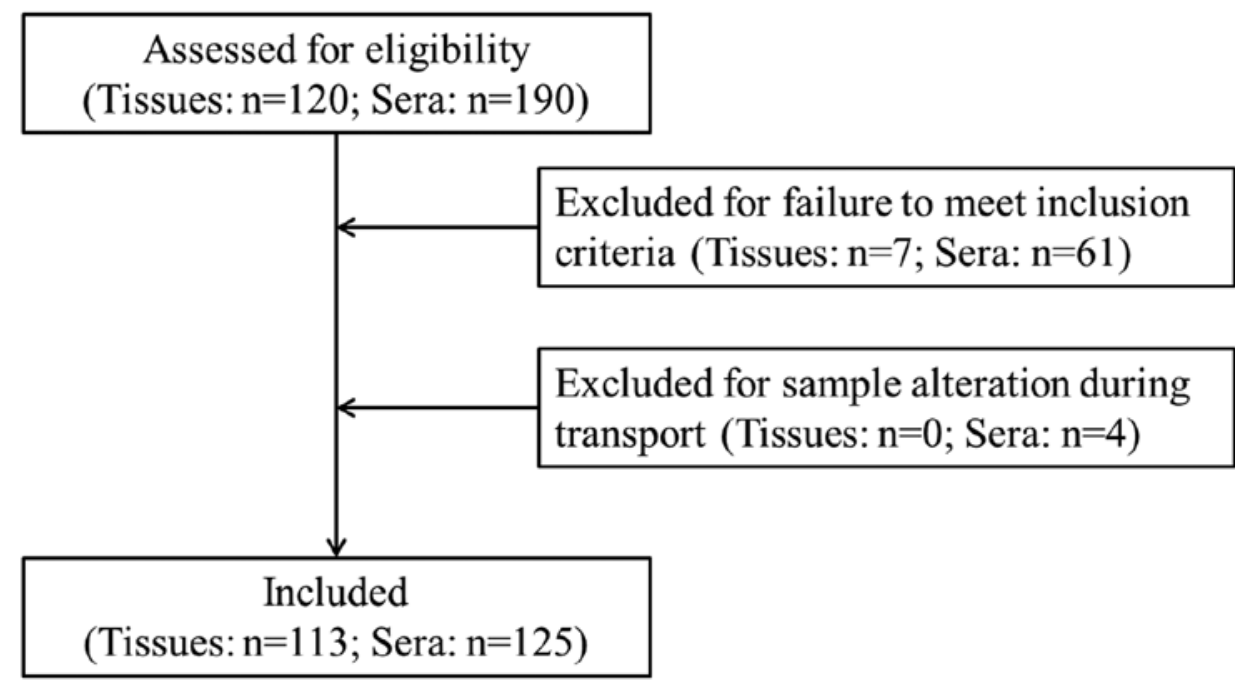

Figure 1. Summary of patient flow diagram. A total of 113 tissue samples and 125 serum samples were included in the present study.

a) RT-qPCR. A $20-\mu 1$ PCR system was used. The total RNA volume in each group was $2 \mu \mathrm{l}$, and $0.8 \mu \mathrm{l}$ of upstream and downstream primers, respectively, was added. A total of $5.6 \mu 1 \mathrm{ddH}_{2} \mathrm{O}, 10 \mu \mathrm{l} 2 \mathrm{X}$ One Step SYBR ${ }^{\circledR}$ RT-PCR Buffer 4 and $0.8 \mu \mathrm{l}$ PrimeScript One Step Enzyme Mix 2 were used. The reaction conditions were as follows: Stage $1,42^{\circ} \mathrm{C}$ for $5 \mathrm{~min}$ and $95^{\circ} \mathrm{C}$ for $10 \mathrm{sec}$; stage $2,95^{\circ} \mathrm{C}$ for $5 \mathrm{sec}$ and $60^{\circ} \mathrm{C}$ for $20 \mathrm{sec}$ (40 cycles). The primer sequences were as follows: ENO1 forward, 5'-GCCTCCTGCTCAAAGTCAAC-3' and reverse, 5'-AACGATGAGACACCATGACG-3'; and $\beta$-actin forward, 5'-CATGTACGTTGCTATCCGAGGC-3' and reverse, 5'-CTC CTTATGACACGACACGAC-3'.

b) Western blot analysis. The cells of the three groups (NC, negative control; si-1, siRNA interference group 1; and si-2, siRNA interference group 2) were lysed in RIPA buffer (RIPA:PMSF, 100:1), and their protein concentration was determined using the bicinchoninic acid assay. Proteins were denatured at $100^{\circ} \mathrm{C}$ for $7 \mathrm{~min}$ and $30 \mu \mathrm{g}$ protein/lane was loaded for SDS-PAGE on a $10 \%$ gel. Subsequently, proteins were transferred onto polyvinylidene difluoride membranes (cat. no. IPVH00010; EMD Millipore). The membranes were blocked with 5\% non-fat milk at room temperature for $2 \mathrm{~h}$ and incubated with primary antibodies against ENO1 $(1: 2,000)$ and $\beta$-actin $(1: 5,000)$ at $4^{\circ} \mathrm{C}$ overnight, followed by HRP-conjugated goat anti-rabbit $\operatorname{IgG}(1: 5,000)$ and anti-mouse secondary antibodies $(1: 6,000)$ at room temperature for $1 \mathrm{~h}$. $\beta$-actin expression was used as a control. The bands were visualized using BeyoECL Plus Enhanced Chemiluminescence reagent (cat. no. P0018FS; Beyotime Institute of Biotechnology). Carestream Molecular Imaging software v5.0 (Carestream Health, Inc.) was used to measure the greyscale density and calculate the relative expression levels.

Detection of cell proliferation using CCK-8. Cells in logarithmic growth phase were used to prepare a cell suspension that was inoculated into 6 -well plates at $\sim 3 \times 10^{5}$ cells/well. Transfection was performed after $24 \mathrm{~h}$ of conventional culture. At $24 \mathrm{~h}$ after transfection, a cell suspension was prepared, and the cells were inoculated into 96-well plates at $\sim 5 \times 10^{5}$ cells/well. At different time points $(24,48$ and $72 \mathrm{~h}$ after transfection), $10 \mu$ l CCK-8 detection solution was added to each well, and the cells were cultured for $2 \mathrm{~h}$. The absorbance at $450 \mathrm{~nm}(\mathrm{OD} 450)$ was measured using a microplate reader. The liver cancer cell proliferation inhibition rate after ENO1-siRNA transfection was calculated using the OD450 and the following formula: Inhibition rate $=(1-$ control group OD450/control group OD450) x100\%.

Assessment of cell migration ability using a scratch test. The experimental liver cancer cells were seeded into a 6-well plate at a density of $8 \times 10^{5}$ cells/well. When the cell density reached $100 \%$, a cross mark was scratched in the middle of the 6-well plate vertically using a disinfected sterile pipette tip $(200 \mu \mathrm{l})$. The cells were repeatedly washed with sterile PBS buffer (6-8 times) until the floating cells were completely removed. The culture medium was replaced with serum-free DMEM, and changes in the scratches were observed and photographed under a microscope at 0,24 and $48 \mathrm{~h}$. The gap size was measured using ImageJ software v1.52a (National Institutes of Health), and the percentage migration was calculated based on the size of the wound at $0 \mathrm{~h}$. The experiment was repeated three times for each group. The effects of different treatment factors on the migration ability of the cells were compared.

Assessment of the migration and invasion ability of cells using a Transwell assay. Transwell inserts (8 $\mu \mathrm{m}, 24-w e l l$ plate; Corning, Inc.) were used to analyse the migration and invasion abilities of liver cancer cell lines. For the invasion assay, the membrane at the bottom of the upper chambers of a Transwell plate was coated with Matrigel. A total of $50 \mu \mathrm{l}$ of $50 \mathrm{mg} / \mathrm{l}$ Matrigel were diluted in serum-free DMEM at a ratio of 1:6, added to each well and incubated at $37^{\circ} \mathrm{C}$ for $30 \mathrm{~min}$. For the migration assay, uncoated Transwell chambers were used. For both the migration and invasion assays, a 600- $\mu$ l cell suspension containing $1.5 \times 10^{5}$ cells in serum-free DMEM was added to the upper chambers of the Transwell, and $600 \mu 1$ DMEM with $10 \%$ FBS was added to the lower chambers. The cells 
A
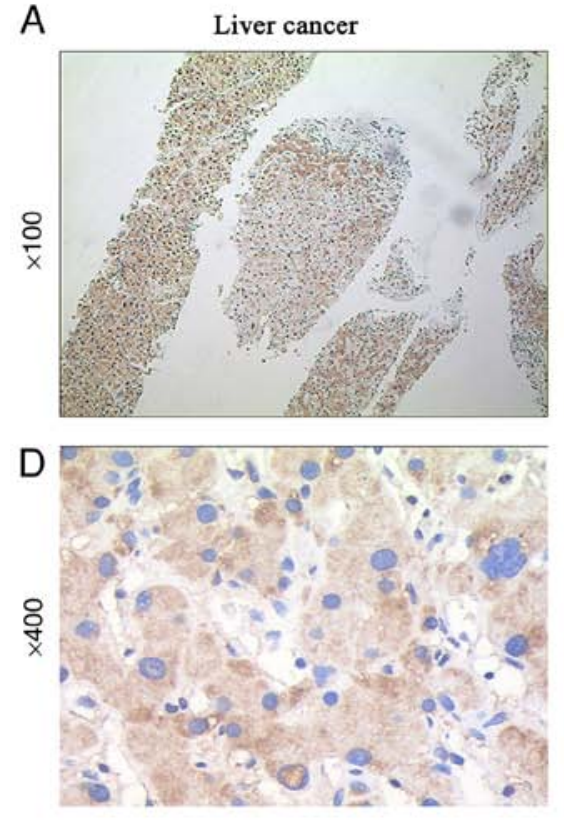

B

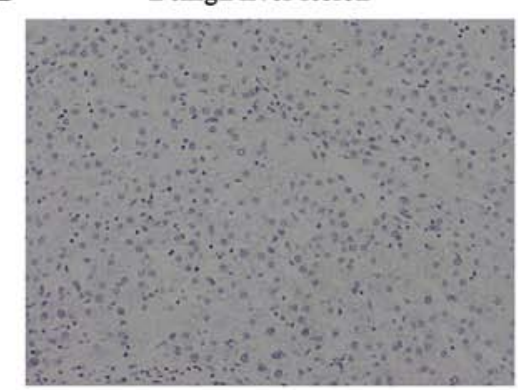

E

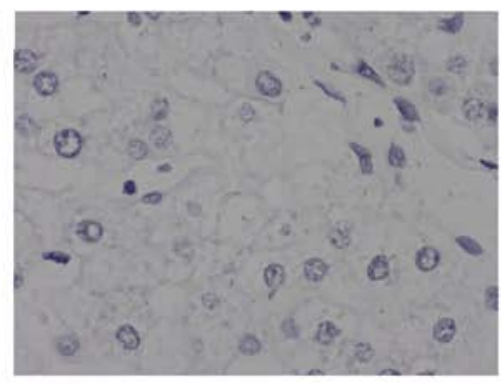

C
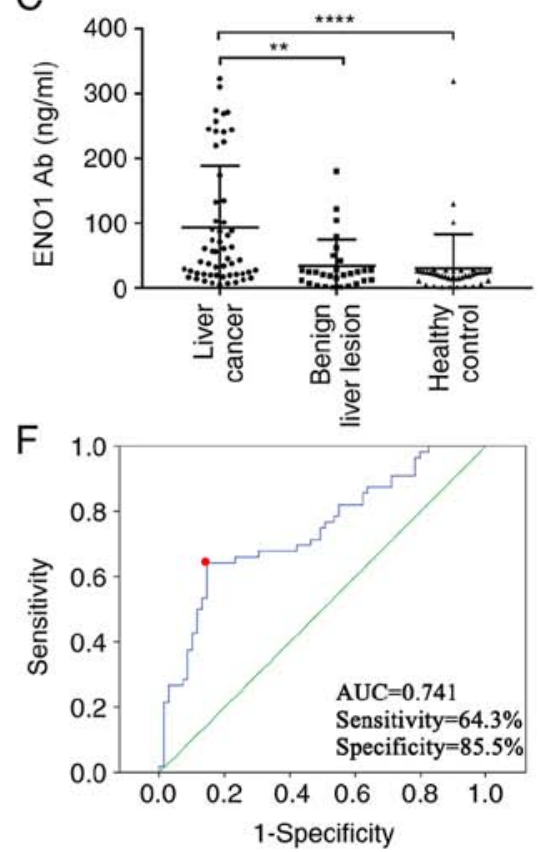

Figure 2. ENO1 expression in liver cancer tissue and peripheral blood of patients with liver cancer. (A) ENO1 expression in liver cancer tissues (x100). (B) ENO1 was not expressed in benign liver lesions (x100). (C) Serum anti-ENO1 antibody levels in patients with liver cancer were higher than those in patients with benign liver lesions and healthy controls. (D) ENO1 expression in liver cancer tissues (x400). (E) ENO1 was not expressed in benign liver lesion (x400). (F) Receiver operating characteristic curve analysis for liver cancer diagnosis using anti-ENO1 antibody levels. The AUC was 0.741, the sensitivity was $64.3 \%$ and the specificity was $85.5 \%$. The red dot represents the cut-off value. ${ }^{* *} \mathrm{P}<0.01 ;{ }^{* * * *} \mathrm{P}<0.0001 \mathrm{ENO}, \alpha$-enolase; AUC, area under the curve.

were routinely cultured for $24 \mathrm{~h}$ (migration assay) or $48 \mathrm{~h}$ (invasion assay), fixed in $4 \%$ paraformaldehyde for $15 \mathrm{~min}$ at room temperature and stained in Giemsa for $15 \mathrm{~min}$ at room temperature. Five fields were randomly selected, and images were captured using an inverted fluorescence microscope (magnification, x400; cat. no. CKX41; Olympus Corporation). The cells were counted using ImageJ software (National Institutes of Health), and each experiment was repeated three times.

Statistical analysis. Statistical analysis was performed using SPSS v19.0 (IBM Corp.) for Windows. The measurement data were presented as the mean $\pm \mathrm{SD}$, or as the median and percentiles $\left(\mathrm{P}_{25}-\mathrm{P}_{75}\right)$. An unpaired Student's t-test was used to determine the differences between two groups. The Kruskal-Wallis test was used to determine the differences among multiple groups, followed by Dunn's test as the post hoc test to determine any significant differences between individual groups. The $\chi^{2}$ test was performed to analyse the association of ENO1 with clinicopathological characteristics. $\mathrm{P}<0.05$ was considered to indicate a statistically significant difference. Receiver operating characteristic (ROC) curve analysis was used to analyze the efficiency of the anti-ENO1 antibody in the diagnosis of liver cancer.

\section{Results}

ENO1 expression in liver cancer tissues. ENO1 expression in tumour tissues (Fig. 2A and D) was primarily localized in the cytoplasm, with some localization also observed in the cell membrane and nucleus. ENO1 expression was not expressed in the majority of the benign liver lesions (Fig. 2B and E). ENO1 expression was observed in all 80 tumour tissues from patients with liver cancer. The expression levels of ENO1 based on the clinical stage, age and sex, were compared among all the groups using the $\mathrm{c}^{2}$ test (Table I). Table I shows that ENO1 expression in liver cancer tissue (43.8\%) was significantly higher than that in benign liver lesions $(15.2 \%)$ $\left(c^{2}=8.356 ; \mathrm{P}=0.005\right)$. ENO1 expression was not associated with clinical stage, age or sex (all $\mathrm{P}>0.05$ ).

Expression of anti-ENOI antibody in the peripheral blood of patients with liver cancer. The levels of anti-ENO1 antibody among the three groups (NC, si-1 and si-2) were compared. The anti-ENO1 antibody concentrations in the three groups showed skewed distributions. Therefore, the levels were expressed using $\mathrm{P}_{50}\left(\mathrm{P}_{25}-\mathrm{P}_{75}\right)$. The anti-ENO1 antibody levels among the three groups were compared using the Kruskal-Wallis test, and Dunn's post hoc test was used for the comparisons between two groups (Fig. 2C and Table II). As presented in Table II, i) the anti-ENO1 antibody levels in the liver cancer group were significantly higher than those in the control and benign liver lesion groups $(\mathrm{P}<0.001)$. The anti-ENO1 antibody levels between the benign liver lesion and control groups were not significantly different $(\mathrm{P}=0.523)$; and ii) the anti-ENO1 antibody levels were not associated with age, sex or clinical stage $(\mathrm{P}=0.835,0.326$ and 0.138 , respectively).

Diagnostic value of the anti-ENO1 antibody level in liver cancer. The ROC curve of liver cancer diagnosis using anti-ENO1 antibody levels was plotted, and the area under the curve (AUC) was calculated. The location with the largest Youden index was used as the best screening cut-off value. The cut-off value for liver cancer diagnosis using the anti-ENO1 antibody level was $29.33 \mathrm{ng} / \mathrm{ml}$; the AUC was 
Table I. ENO1 expression in pathological tissues.

\begin{tabular}{|c|c|c|c|c|c|c|}
\hline \multirow[b]{2}{*}{ Group } & \multirow[b]{2}{*}{ No. of cases } & \multicolumn{2}{|c|}{ ENO1 expression } & \multirow[b]{2}{*}{ Positive rate, $\%$} & \multirow[b]{2}{*}{$\chi^{2}$} & \multirow[b]{2}{*}{ P-value } \\
\hline & & Positive & Negative & & & \\
\hline Benign lesion tissue & 33 & 5 & 28 & 15.2 & 8.356 & 0.005 \\
\hline Liver cancer tissue & 80 & 35 & 45 & 43.8 & & \\
\hline Clinical stage & & & & & 0.250 & 0.157 \\
\hline $\mathrm{I}+\mathrm{II}$ & 37 & 21 & 16 & 56.8 & & \\
\hline III & 43 & 22 & 21 & 51.2 & & \\
\hline Age, years & & & & & 0.033 & 0.525 \\
\hline$>60$ & 54 & 24 & 30 & 44.4 & & \\
\hline$\leq 60$ & 26 & 11 & 15 & 42.3 & & \\
\hline Sex & & & & & 1.270 & 0.276 \\
\hline Male & 64 & 26 & 38 & 40.6 & & \\
\hline Female & 16 & 9 & 7 & 56.3 & & \\
\hline
\end{tabular}

ENO1, $\alpha$-enolase.

Table II. Comparison of the serum anti-ENO1 antibody levels among the three groups of participants [P50 (P25-P75)].

\begin{tabular}{lccr}
\hline Group & No. of cases & Anti-ENO1 antibody, ng/ml & P-value \\
\hline Liver cancer & 56 & $50.88(21.67-133.97)$ & $<0.001^{\mathrm{a}}$ \\
Age, years & & & 0.835 \\
$>60$ & 23 & $57.42(21.57-90.44)$ & 0.326 \\
$\leq 60$ & 33 & $45.01(21.08-197.08)$ & \\
Sex & & & \\
Male & 38 & $50.88(22.41-225.12)$ & 0.138 \\
Female & 18 & $45.77(20.15-100.98)$ & \\
Clinical stage & & & \\
I+II & 27 & $34.12(20.13-118.08)$ & $0.523^{\mathrm{b}}$ \\
III & 29 & $22.94(10.02-35.21)$ & \\
Benign liver lesion & 29 & $21.46(12.83-26.90)$ & \\
Healthy control & 40 & 5 & \\
\hline
\end{tabular}

${ }^{a}$ The serum anti-ENO1 antibody levels in the liver cancer group were significantly higher than those in the control and benign liver lesion groups; ${ }^{b}$ The anti-ENO1 antibody levels in the benign liver lesion and control groups were not significantly different. ENO1, $\alpha$-enolase.

0.741 , the sensitivity was $64.3 \%$, and the specificity was $85.5 \%$ (Fig. 2F).

\section{Validation of the interference effect}

$R T-q P C R$. The relative expression levels of the ENO1 gene in the siRNA groups, si-1 and si-2, were both significantly lower than those in the control group. Compared with that in the control group, the ENO1 interference rate in HepG2 cells reached $85.3 \%$ in the si- 1 group and $55.0 \%$ in the si-2 group. The interference rate in Huh7 cells reached $82.8 \%$ in the si- 1 group and $88.4 \%$ in the si-2 group (Fig. 3A and B).

Western blot analysis. The western blot analysis results are presented in Fig. 3C and D. The ENO1 expression levels were normalized to $\beta$-actin expression levels. The results showed that the relative expression levels of the ENO1 protein in HepG2 and Huh7 cells in the si-1 and si-2 groups were significantly lower than those in the control group $(\mathrm{P}<0.0001)$.

Effect of ENO1 interference on the proliferation of liver cancer cells. The results of the CCK-8 assay showed that, compared with the NC group, ENO1 siRNA treatment resulted in different degrees of HepG2 and Huh7 cell proliferation inhibition after $72 \mathrm{~h}$ of transfection, and the differences were statistically significant as analysed by Kruskal-Wallis test (Fig. 4).

Effect of ENO1 interference on the migration ability of liver cancer cells. The results of the scratch tests showed that, 
A

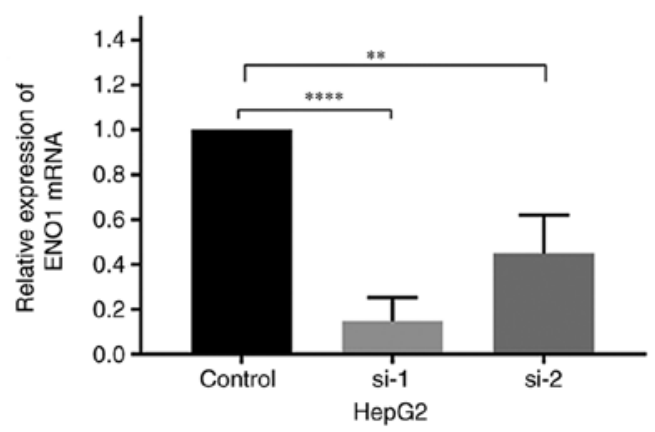

B

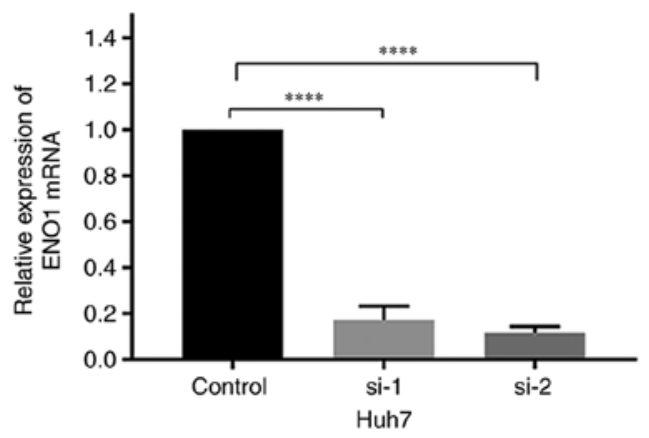

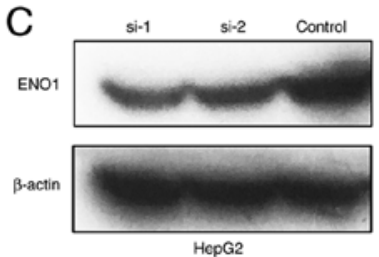
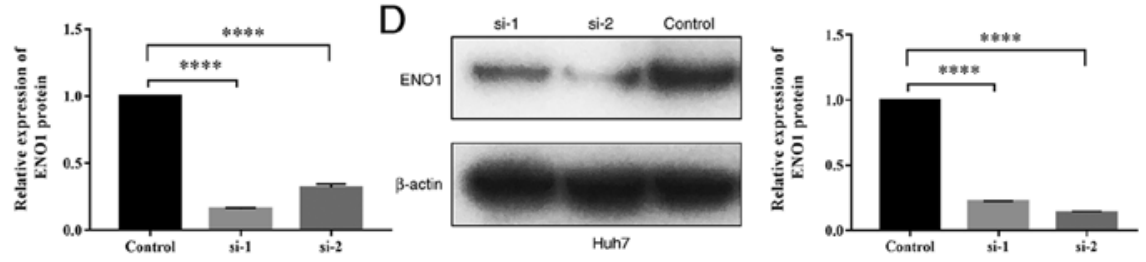

Figure 3. Validation of the siRNA interference effect on ENO1 expression in liver cancer cells. (A) RT-qPCR validation of the effect of siRNA interference on ENO1 expression in HepG2 cells. The results showed that the relative expression levels of the ENO1 gene in the si-1 and si-2 groups were lower than those in the control group. (B) RT-qPCR validation of the effect of siRNA interference on ENO1 expression in Huh7 cells. The results showed that the relative expression levels of the ENO1 gene in the si-1 and si-2 groups were lower than those in the control group. (C) Western blot validation of the effect of siRNA interference on ENO1 expression in HepG2 cells. The results showed that the relative expression levels of the ENO1 protein in HepG2 cells in the si-1 and si-2 groups were significantly lower than those in the control group. (D) Western blot validation of the effect of siRNA interference on ENO1 expression in Huh7 cells. The results showed that the relative expression levels of the ENO1 protein in Huh7 cells in the si-1 and si-2 groups were significantly lower than those in the control group. ${ }^{* *} \mathrm{P}<0.01 ;{ }^{* * * * *} \mathrm{P}<0.0001$. siRNA, small interfering RNA; ENO1, $\alpha$-enolase; si-1, siRNA interference group 1; si-2, siRNA interference group 2; RT-qPCR, reverse transcription-quantitative PCR.
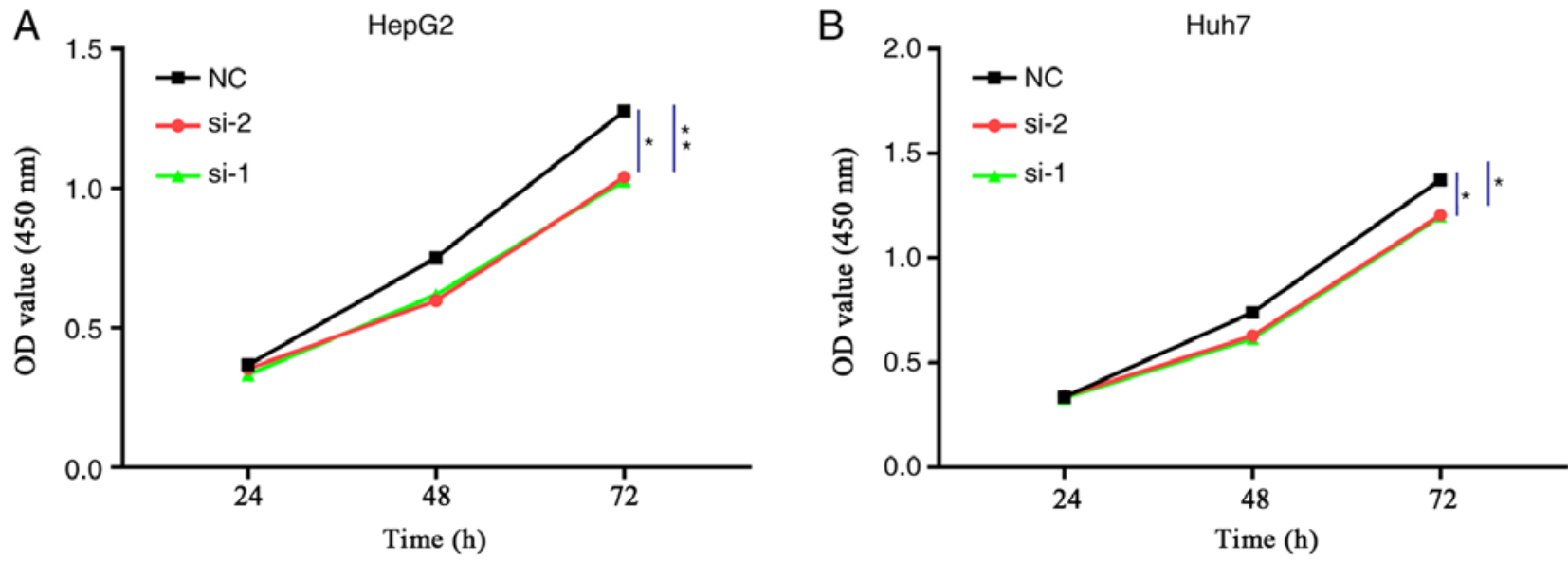

Figure 4. Effect of ENO1 siRNA on liver cancer cell proliferation. (A) HepG2 and (B) Huh7 cell proliferation was suppressed after ENO1 siRNA treatment. Results show that $72 \mathrm{~h}$ after transfection in the si-1 and si-2 groups, ENO1 siRNA treatment resulted in proliferation inhibition in HepG2 and Huh7 cells. In HepG2 cells, the differences in cell proliferation between the NC and the siRNA-treated groups were statistically significant $(\mathrm{P}<0.05$ vs. si-2 group and $\mathrm{P}<0.01$ vs. si-1 group). Cell proliferation in Huh7 cells was also significantly different. ${ }^{*} \mathrm{P}<0.05 ;{ }^{* *} \mathrm{P}<0.01$. ENO1, $\alpha$-enolase; siRNA, small interfering RNA; si-1, siRNA interference group 1; si-2, siRNA interference group 2; OD, optical density; NC, negative control.

compared with the NC group, HepG2 and Huh7 cell migration after ENO1 siRNA treatment became slower, and the differences were statistically significant by Student's t-test at $48 \mathrm{~h}$ after treatment (Fig. 5).

Effect of ENO1 downregulation on the invasion ability of liver cancer cells. The results of the Transwell experiment showed that, compared with the NC group, the in vitro invasion and migration abilities of HepG2 and Huh7 cells after ENO1 siRNA treatment significantly decreased (Fig. 6).

\section{Discussion}

ENO1, also known as $\alpha$-enolase, is one of the three subtypes of enolase. ENO1 is mainly present in the cytoplasm, but also in the membrane and nucleus of a variety of eukaryotic cells. This enzyme has important functions in cellular energy metabolic processes. ENO1 converts 2-phosphoglycerate into phosphoenolpyruvate during glycolysis and can also catalyse the reverse reaction to convert phosphoenolpyruvate into 2-phosphoglycerate (10). In recent years, the association of 
A

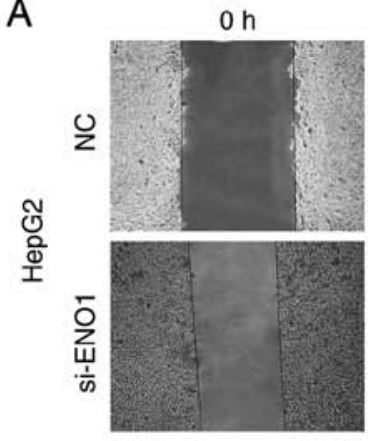

C

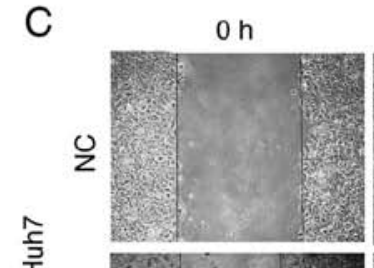

독

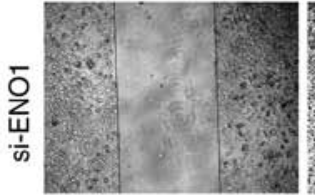

$24 \mathrm{~h}$
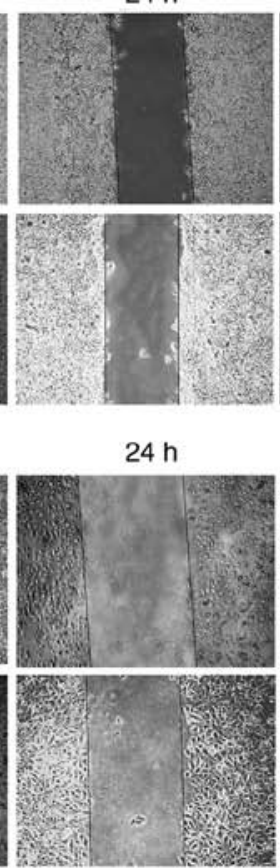

$48 \mathrm{~h}$
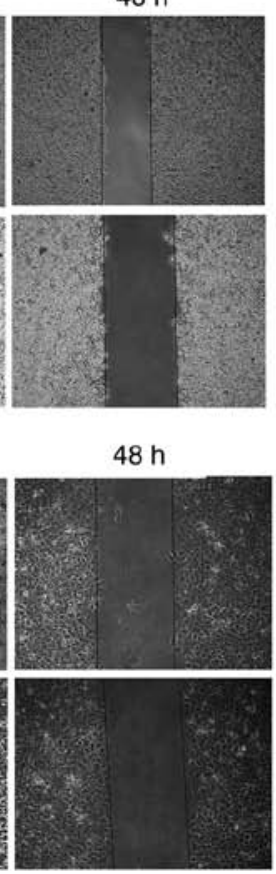

B

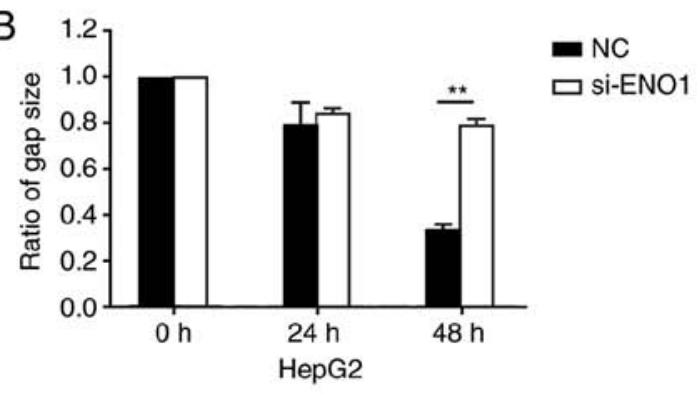

D

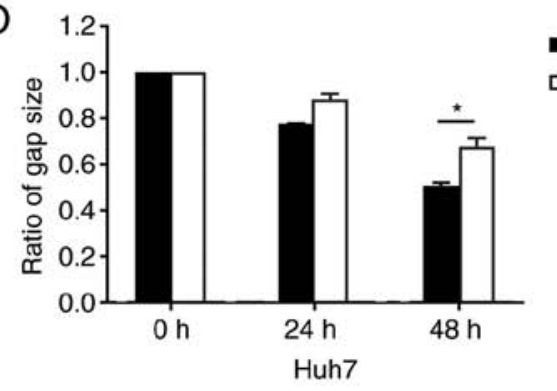

Figure 5. Effect of ENO1 siRNA on the migration ability of liver cancer cells. (A) Compared with that of the NC group, HepG2 cell migration after ENO1 siRNA treatment became slower in the si-1 and si-2 groups, and the difference was statistically significant at $48 \mathrm{~h}$ after treatment. (B) Gap size was measured using ImageJ software and the ratio of gap size after ENO1 siRNA treatment in HepG2 cells was calculated based on the size of the wound at $0 \mathrm{~h}$. (C) Compared with that of the NC group, Huh7 cell migration after ENO1 siRNA treatment became slower in the si-1 and si-2 groups, and the difference was statistically significant at $48 \mathrm{~h}$ after treatment. (D) Gap size was measured with the ImageJ software and the ratio of gap size after ENO1 siRNA treatment in Huh7 cell was calculated based on the size of the wound at $0 \mathrm{~h} .{ }^{*} \mathrm{P}<0.05 ;{ }^{* *} \mathrm{P}<0.01$. ENO1, $\alpha$-enolase; siRNA, small interfering RNA; NC, negative control; si-1, siRNA interference group $1 ;$ si-2, siRNA interference group 2.

A
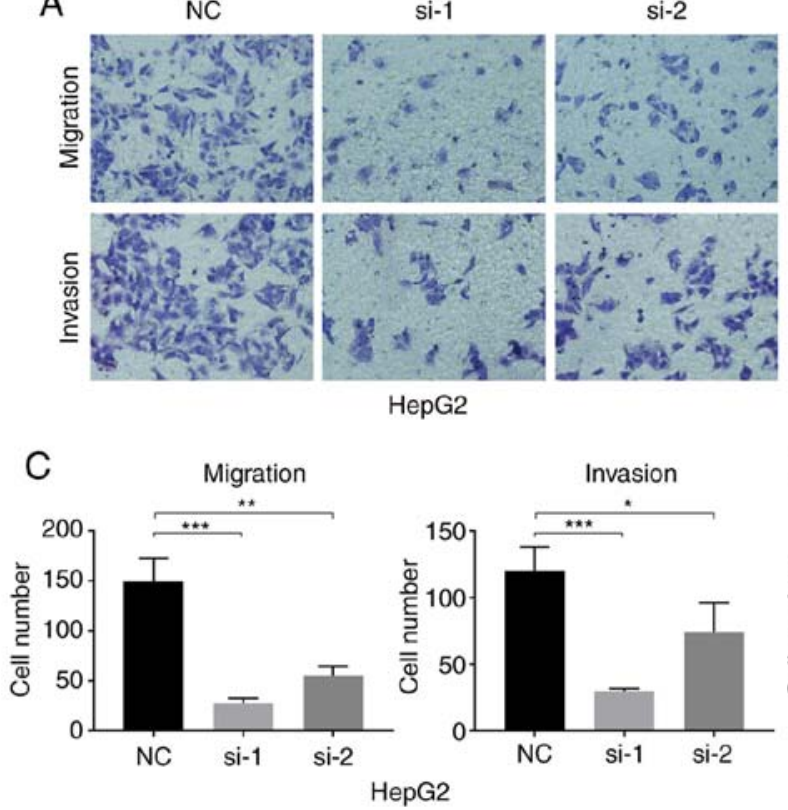

B NC

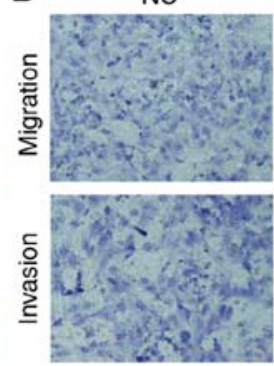

D

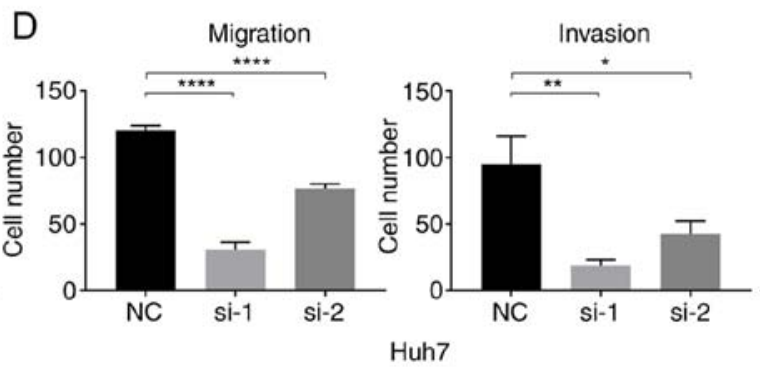

Figure 6. Effect of ENO1 siRNA on the invasion and migration abilities of liver cancer cells. Compared with the NC group, the in vitro invasion and migration abilities of (A) HepG2 and (B) Huh7 cells after ENO1 siRNA treatment decreased in the si-1 and si-2 groups, and the differences were statistically significant. Numbers of (C) HepG2 and (D) Huh7 cells were measured with the ImageJ software after ENO1 siRNA treatment. ${ }^{*} \mathrm{P}<0.05$; ${ }^{* *} \mathrm{P}<0.01$; ${ }^{* * *} \mathrm{P}<0.001$; ${ }^{* * * * *} \mathrm{P}<0.0001$. ENO1, $\alpha$-enolase; siRNA, small interfering RNA; NC, negative control; si-1, siRNA interference group 1; si-2, siRNA interference group 2.

ENO1 with malignant tumours has received increasing attention (11-13). A number of studies have observed higher levels of ENO1 in cell lines and in vivo tissues of malignant tumours, including pancreatic cancer, renal cell carcinoma and glioma, suggesting that ENO1 has tumour growth-promoting functions (14-16). The main mechanism underlying the high 
ENO1 expression in tumour tissues is associated with the Warburg effect proposed by Dr Otto Warburg in 1956 (17). Warburg hypothesized that the difference in energy source was the main reason for the higher growth rate of tumour cells compared with that of normal cells. Under sufficient oxygen conditions, tumour cells utilize glucose mainly through the aerobic glycolysis pathway, and reduce aerobic phosphorylation in mitochondria, which is considered to be the most important cause of tumour development. A study by Altenberg and Greulich (18) also confirmed that the genes of glycolysis enzymes are ubiquitously overexpressed in tumour cells. Altenberg and Greulich considered that the overexpression of glycolysis enzymes might be a key factor causing excessive tumour cell proliferation. Therefore, as an important glycolytic enzyme, ENO1 might play an important role in the development, progression and metastasis of malignant tumours.

A previous study by our group showed that ENO1 was more highly expressed in lung cancer than in benign tumours; additionally, the expression levels of serum ENO1 antibodies had a diagnostic value in lung cancer (19). However, the role of ENO1 in the development of liver cancer remains unclear and, to the best of our knowledge, no studies have investigated the expression levels of ENO1 antibodies in the peripheral blood and their diagnostic value in liver cancer.

To further elucidate the role of ENO1 in liver cancer invasion and metastasis, HepG2 and Huh7 liver cancer cells were treated with ENO1 siRNA in the present study. The interference effect was validated by RT-qPCR and western blot analysis. The proliferation ability of liver cancer cells was measured using the CCK- 8 assay; the migration ability of liver cancer cells was measured using a scratch experiment; and the invasion and migration abilities of liver cancer cells were measured using a Transwell assay. The results revealed that, after ENO1 gene interference, the proliferation, migration and invasion abilities of HepG2 and Huh7 liver cancer cells exhibited different degrees of suppression, suggesting that ENO1 promotes liver cancer growth and metastasis. The expression of ENO1 protein in liver cancer tissue and benign liver lesions was also measured using immunohistochemistry. The results showed that the ENO1 protein expression in liver cancer tissues $(43.8 \%)$ was significantly higher than that in benign liver lesions (15.2\%), which was consistent with the results of a previous study (9). In the present study, no obvious association between ENO1 and clinical parameters (stage, age or sex) was observed. However, Zhu et al (9) suggested that ENO1 is associated with Tumor Node Metastasis stage, which should be further investigated using a larger sample size.

In the glycolysis pathway, a number of metabolic enzymes, including ENO1, can be secreted from cells through the exosome release pathway (20). Furthermore, with ENO1 overexpression in tumour cells, ENO1 may be released into peripheral blood through tumour cell apoptosis and necrosis or other non-classical pathways (21).

However, the levels of ENO1 protein in the serum of patients with liver cancer and of patients with benign liver lesions were not significantly different in the present study, which was inconsistent with the results of a previous study (22). A reason for this inconsistency may be that ENO1 expression is unstable and easy to degrade in the serum environment; therefore, future studies should perform experiments to further explore and confirm this explanation. However, a high level of anti-ENO1 antibody in the serum of patients with liver cancer was detected. The reasons are as follows: As a tumour-associated antigen, ENO1 can stimulate the immune system in the body to produce specific anti-ENO1 autoantibodies. However, the mechanism underlying the production of autoantibodies remains unclear. It is possible that aberrant tumour cells continue to die, causing sustained exposure of intracellular proteins after post-translational modification or remodelling, and that a variation in tumour cells stimulates the immune system to induce an immune response to produce autoantibodies (23). The current tumour immunity theory suggests that this immune response occurs in early-stage tumours, and that the produced autoantibody has a more stable titre in peripheral blood, suggesting that this autoantibody might be used as a tumour-associated serum marker. The serum anti-ENO1 antibody levels in patients with liver cancer, patients with benign liver disease and healthy controls were measured using ELISA in the present study. The results showed that the levels of anti-ENO1 antibody in the liver cancer group were significantly higher than those in the benign liver lesion and control groups, whereas there was no significant difference between the benign liver lesion and healthy control groups. The present study also analysed the association of the anti-ENO1 antibody level with age, sex and clinical stage. The results revealed that the level of anti-ENO1 antibody was not associated with any of these factors. ROC curve analysis showed that liver cancer diagnosis using anti-ENO1 antibody levels had a sensitivity of $64.3 \%$ and a specificity of $85.5 \%$, suggesting that liver cancer diagnosis using anti-ENO1 antibody levels has a certain accuracy, and that patients have a certain titre in peripheral blood in early-stage liver cancer. However, a limitation of this study was the small sample size. Therefore, the conclusions need to be further validated with a larger sample size.

In conclusion, the results of the present study indicated that ENO1 can promote liver cancer invasion and migration. The ENO1 protein expression level in tissues and the anti-ENO1 antibody level in peripheral blood have important value for the diagnosis of liver cancer, and can be used as potential liver cancer-associated markers. Serum anti-ENO1 antibody detection has the advantages of convenient sample collection and minimal trauma. Therefore, the value of anti-ENO1 antibodies in liver cancer diagnosis may be greater than that of tissue protein expression. However, there are some limitations in the present study. The ability of invasion and migration of liver cancer cells was only verified to decrease after interference of ENO1; however, the changes after upregulation of ENO1 were not investigated, and proliferation and invasion-related biomarkers were not determined. The focus of our future research will be the molecular mechanism of ENO1 that promotes liver cancer invasion and migration, as well as relevant targets for the treatment of liver cancer.

\section{Acknowledgements}

Not applicable. 


\section{Funding}

The present study was supported by the Basic Public Welfare Research Project of Zhejiang Province (grant no. LGF19H200004), the Medical and Scientific Research Project of Zhejiang Province (grant no. 2020KY326) and the Science and Technology Planning Project of Shaoxing (grant no. 2018C30057).

\section{Availability of data and materials}

The datasets used and/or analysed during the present study are available from the corresponding author on reasonable request.

\section{Authors' contributions}

LZ and LH conceived the study and wrote the manuscript. LZ, LH and TL performed the experiments. YY assisted with the collection and analysis of the patient data regarding the clinicopathological parameters of liver cancer. All authors read and approved the final manuscript.

\section{Ethics approval and consent to participate}

The study was approved by the Ethics Committee of Shaoxing People's Hospital (Shaoxing, China), and signed informed consent was provided by the patients.

\section{Patient consent for publication}

Not applicable.

\section{Competing interests}

The authors declare that they have no competing interests.

\section{References}

1. Bray F, Ferlay J, Soerjomataram I, Siegel RL, Torre LA and Jemal A: Global cancer statistics 2018: GLOBOCAN estimates of incidence and mortality worldwide for 36 cancers in 185 countries. CA Cancer J Clin 68: 394-424, 2018.

2. Gingold JA, Zhu D, Lee DF, Kaseb A and Chen J: Genomic profiling and metabolic homeostasis in primary liver cancers. Trends Mol Med 24: 395-411, 2018.

3. Youssef AA, Issa HA, Omar MZ, Behiry EG, Elfallah AA, Hasaneen A, Darwish M and Ibrahim DB: Serum human endothelia cell-specific molecule-1 (endocan) and vascular endothelial growth factor in cirrhotic $\mathrm{HCV}$ patients with hepatocellular carcinoma as predictors of mortality. Clin Exp Gastroenterol 11: 431-438, 2018.

4. Zhang Z,Zhang Y, Wang Y,Xu L and Xu W: Alpha-fetoprotein-L3 and Golgi protein 73 may serve as candidate biomarkers for diagnosing alpha-fetoprotein-negative hepatocellular carcinoma. Onco Targets Ther 9: 123-129, 2015.

5. Park SJ, Jang JY, Jeong SW, Cho YK, Lee SH, Kim SG, Cha SW, Kim YS, Cho YD, Kim HS, et al: Usefulness of AFP, AFP-L3, and PIVKA-II, and their combinations in diagnosing hepatocellular carcinoma. Medicine (Baltimore) 96: e5811, 2017.
6. Principe M, Borgoni S, Cascione M, Chattaragada MS, Ferri-Borgogno S, Capello $M$, Bulfamante S, Chapelle J, Di Modugno F, Defilippi P, et al: Alpha-enolase (ENO1) controls alpha v/beta 3 integrin expression and regulates pancreatic cancer adhesion, invasion, and metastasis. J Hematol Oncol 10: $16,2017$.

7. Huang Z, Lin B, Pan H, Du J, He R, Zhang S and Ouyang P: Gene expression profile analysis of ENO1 knockdown in gastric cancer cell line MGC-803. Oncol Lett 17: 3881-3889, 2019.

8. Sun L, Lu T, Tian K, Zhou D, Yuan J, Wang X, Zhu Z, Wan D, Yao Y, Zhu X, et al: Alpha-enolase promotes gastric cancer cell proliferation and metastasis via regulating AKT signaling pathway. Eur J Pharmacol 845: 8-15, 2019.

9. Zhu W, Li H, Yu Y, Chen J, Chen X, Ren F, Ren Z and Cui G: Enolase-1 serves as a biomarker of diagnosis and prognosis in hepatocellular carcinoma patients. Cancer Manag Res 10: 5735-5745, 2018

10. Zhan P, Wang Y, Zhao S, Liu C, Wang Y, Wen M, Mao JH, Wei $G$ and Zhang P: FBXW7 negatively regulates ENO1 expression and function in colorectal cancer. Lab Invest 95: 995-1004, 2015.

11. Ryans K, Omosun Y, McKeithen DN, Simoneaux T, Mills CC, Bowen N, Eko FO, Black CM, Igietseme JU and He Q: The immunoregulatory role of alpha enolase in dendritic cell function during Chlamydia infection. BMC Immunol 18: 27, 2017.

12. Yin $\mathrm{H}$, Wang $\mathrm{L}$ and Liu HL: ENO1 overexpression in pancreatic cancer patients and its clinical and diagnostic significance. Gastroenterol Res Pract 2018: 3842198, 2018.

13. Yu S, Li N, Huang Z, Chen R, Yi P, Kang R, Tang D, Hu X and Fan X: A novel lncRNA, TCONS_00006195, represses hepatocellular carcinoma progression by inhibiting enzymatic activity of ENO1. Cell Death Dis 9: 1184, 2018.

14. Sun L, Guo C, Cao J, Burnett J, Yang Z, Ran Y and Sun D: Over-expression of alpha-enolase as a prognostic biomarker in patients with pancreatic cancer. Int J Med Sci 14: 655-661, 2017.

15. White-Al Habeeb NM, Di Meo A, Scorilas A, Rotondo F, Masui O, Seivwright A, Gabril M, Girgis AH, Jewett MA and Yousef GM: Alpha-enolase is a potential prognostic marker in clear cell renal cell carcinoma. Clin Exp Metastasis 32: 531-541, 2015.

16. Song Y, Luo Q, Long H, Hu Z, Que T, Zhang X, Li Z, Wang G, Yi L, Liu Z, et al: Correction: Alpha-enolase as a potential cancer prognostic marker promotes cell growth, migration, and invasion in glioma. Mol Cancer 13: 235, 2015.

17. Warburg O: On the origin of cancer cells. Science 123: 309-314, 1956.

18. Altenberg B and Greulich KO: Genes of glycolysis are ubiquitously overexpressed in 24 cancer classes. Genomics 84: 1014-1020, 2004.

19. Zhang L, Wang H and Dong X: Diagnostic value of $\alpha$-enolase expression and serum $\alpha$-enolase autoantibody levels in lung cancer. J Bras Pneumol 44: 18-23, 2018.

20. Mathivanan S and Simpson RJ: ExoCarta: A compendium of exosomal proteins and RNA. Proteomics 9: 4997-5000, 2009.

21. Mazurek S, Boschek CB, Hugo F and Eigenbrodt E: Pyruvate kinase type $\mathrm{M} 2$ and its role in tumor growth and spreading. Semin Cancer Biol 15: 300-308, 2005.

22. Luo X, Wei Y, Hai L, Hu Y, Zhao Z, Ma W, Ma L, Liu X and Ding X: Preliminary study on serum marker $\alpha$-enolase of liver cancer in the diagnosis of liver cancer. Chin J Hepatol 27: 505-510, 2019 (In Chinese).

23. Tan HT, Low J, Lim SG and Chung MC: Serum autoantibodies as biomarkers for early cancer detection. FEBS J 276: 6880-6904, 2009.

This work is licensed under a Creative Commons Attribution-NonCommercial-NoDerivatives $\quad 4.0$ International (CC BY-NC-ND 4.0) License. 